\title{
Value of heart rate variability on dynamic electrocardiogram in predicting ventricular fibrillation in elderly acute myocardial infarction patients
}

\author{
Yuan Zhang ${ }^{1}$, Jing Wang ${ }^{1}$, Yonggang $\mathrm{Xu}^{2}$ \\ ${ }^{1}$ ECG Room, Liaocheng People’s Hospital, Liaocheng, China; ${ }^{2}$ Bachelor of Medicine, Department of Cardiology, Liaocheng People's Hospital, \\ Liaocheng, China \\ Contributions: (I) Conception and design: J Wang; (II) Administrative support: J Wang; (III) Provision of study materials or patients: Y Xu, Y Zhang; \\ (IV) Collection and assembly of data: Y Xu, Y Zhang; (V) Data analysis and interpretation: Y Zhang; (VI) Manuscript writing: All authors; (VII) Final \\ approval of manuscript: All authors. \\ Correspondence to: Jing Wang. ECG Room, Liaocheng People’s Hospital, Liaocheng, China. Email: wj18663506828@126.com.
}

\begin{abstract}
Background: Ventricular fibrillation (VF) is the main cause of sudden death in Acute myocardial infarction (AMI) patients. Identifying arrhythmia as early as possible is of great significance in improving prognosis of AMI patients. The article is aimed at exploring the predictive value of heart rate variability (HRV) of dynamic electrocardiogram (DCG) in VF of elderly AMI patients.

Methods: A total of 122 elderly AMI patients were enrolled as the research objects. They were divided into VF group and simple AMI group (AMI group). All underwent DCG test after admission. The basic data of patients were compared. HRV of DCG was observed. The predictive value of HRV of DCG in VF of elderly AMI patients was evaluated by receiver operating characteristic (ROC) curves.

Results: The ratio of Killip at grade II-III, activity of creatine kinase-MB (CK-MB), standard deviation of all R-R intervals (SDNN), the standard deviation of the R-R intervals (SDANN), root-mean-square of the successive differences of normal RR intervals (rMSSD) and percentage of NN50 in the total number of NN intervals PNN50 (PNN50\%)]in VF group were higher than those in $\mathrm{AMI}$ group $(\mathrm{P}<0.05)$. The area under the ROC curves (AUC)and cut-off values of SDNN, SDANN, rMSSD and PNN50\% were $(0.773,0.761$, $0.800,0.819)$ and $(135.675,106.850,33.570,14.080)$, respectively.
\end{abstract}

Conclusions: HRV of DCG is of certain predictive value for VF in the elderly with AMI, which is expected to be an auxiliary diagnosis method of $\mathrm{VF}$.

Keywords: Acute myocardial infarction; Elderly patient; Ventricular fibrillation; Dynamic electrocardiogram; Heart rate variability; Predictive value

Submitted Apr 23, 2020. Accepted for publication Sep 16, 2020.

doi: 10.21037/apm-20-1362

View this article at: http://dx.doi.org/10.21037/apm-20-1362

\section{Introduction}

Acute myocardial infarction (AMI) is the acute thrombotic occlusion of coronary arteries caused by the rupture of atherosclerotic plaque in the coronary arteries. It results in interruption of blood flow and myocardial ischemia, and long-term ischemia will lead to myocardial ischemic injury (1). Before the onset of AMI, some patients may have the clinical symptoms (such as palpitations, shortness of breath, chest discomfort, and/or precordial pain) of coronary heart disease. Its typical symptom is the precordial pain, which occurs mainly in the posterior side of sternum but may extend to the left ribs or to the shoulders, back, oral area, or head. In most cases the pain can last for more than 30 minutes and will not be quickly resolved even under a resting state. Many factors may trigger AMI. Any factor that can cause thrombosis, intravascular plaque shedding, 
and coronary artery stenosis or spasm can induce the onset of AMI. Daily factors such as smoking, alcoholism, mood swings, overeating, and overwork can also increase the risk of AMI. Early detection and early treatment are particularly important for AMI. The main therapies include thrombolytic therapy, percutaneous coronary intervention, and emergency bypass surgeries, which may help to reduce the area of myocardial infarction, alleviate myocardial injury, and protect myocardial function in patients with AMI. Adopting rehabilitation measures (including appropriate exercise) and avoiding mood swings are also important for the patients after discharge.

Ventricular fibrillation (VF) is the main cause of sudden death in AMI patients, and timely electrical defibrillation is the mainstay of treatment for AMI (2). The prevalence of AMI has gradually increased along with the aging of China's population, as has the incidence of AMI complicated by VF. Reperfusion therapy can reduce myocardial injury, reduce infarct size, decrease the severity of myocardial ischemia, improve the cardiac pumping function, and increase the patient's long-term survival (3). However, severe ventricular arrhythmia remains a major cause of death in AMI patients. Therefore, early identification and timely intervention of ventricular arrhythmias in AMI patients is valuable for lowering the risk of AMI death. Dynamic electrocardiogram (DCG, also known as Holter monitoring) is a method which can continuously record and analyze the changes in the electrocardiogram (ECG) of the human heart in an active and quiet state for an extended period. By recording all the abnormal waves, it can offer accurate evidence for the diagnosis of a variety of heart diseases (4). Heart rate variability (HRV) refers to the fluctuations between consecutive heartbeat cycles. Regulated by neurohumoral factors, it can reflect the changes in sympathovagal balance and is valuable for the diagnosis and prognosis of heart diseases (5). The change of heart rate can reflect cardiac autonomic activity, and HRV an quantify its evaluation (6). Song et al. (6) found that HRV-related parameters were highly predictive for cardiac mortality after AMI. In our current study, we selected 122 elderly AMI patients admitted to our hospital from January 2018 to January 2020 as the subjects, and the predictive value of HRV for elderly inpatients with AMI complicated by VF was assessed. We hope the findings produced can help provide new evidence for early clinical diagnosis.

We present the following article in accordance with the STROBE reporting checklist (available at http://dx.doi. org/10.21037/apm-20-1362).

\section{Methods}

\section{Clinical data}

A total of 122 elderly AMI patients admitted to our hospital from January 2018 to January 2020 were selected as the subjects. The inclusion criteria were as follows: (I) aged $\geq 60$ years; (II) meeting the diagnostic criteria of AMI (7): (i) with a history of ischemic chest pain; (ii) with a dynamic change on the electrocardiogram; and (iii) with a dynamic change in the concentrations of serum myocardial markers related to myocardial necrosis (at least two of these three criteria are met); (III) duration of ischemic chest pain $\geq 30 \mathrm{~min}$; (IV) ST segment elevation in two or more anatomically contiguous chest leads; (V) interval from symptom onset to admission $<6 \mathrm{~h}$; (VI) no pacemaker treatment during DCG monitoring; and (VII) concentration of serum biochemical markers in the patient's heart outside of the clinical reference range (8). The exclusion criteria were as follows: (I) died within $24 \mathrm{~h}$ after admission; (II) with old myocardial infraction (OMI) and/or other organic heart disease; (III) with accompanying malignant tumors and/or blood diseases; (IV) with abnormal liver/kidney function; (V) with sick sinus syndrome, using cardiac pacemaker due to sinus arrest, and with atrial flutter or other non-sinus rhythms; (VI) with autonomic dysfunction; and/or (VII) with psychiatric disorders. All the patients attended the study voluntarily. The study was conducted in accordance with the Declaration of Helsinki (as revised in 2013). Patients and their families signed informed consent documents. The study was approved by the ethics committee of our hospital (No. 2018010).

\section{Study methods}

Patients were divided into a VF group (with the presence of VF) and a simple AMI group (without the presence of VF). The clinical data, laboratory test results, and HRV indicators of these two groups were compared. The diagnostic criteria of VF (9) included: The electrocardiography revealed tremor waves irregular in morphology, amplitude, and interval, and the QRS complex could not be distinguished from $\mathrm{T}$ wave; hemodynamic changes were also observed. The dynamic electrocardiographic changes were recorded within 24 $\mathrm{h}$ after admission by using the DMS300-4A dynamic ECG analysis system (Dim Corporation, USA). HRV time-domain indexes including standard deviation (SD) of 5-minute RR intervals (SDANN), standard deviation of all R-R intervals (SDNN), root-mean-square of the 
Table 1 General data of the two groups

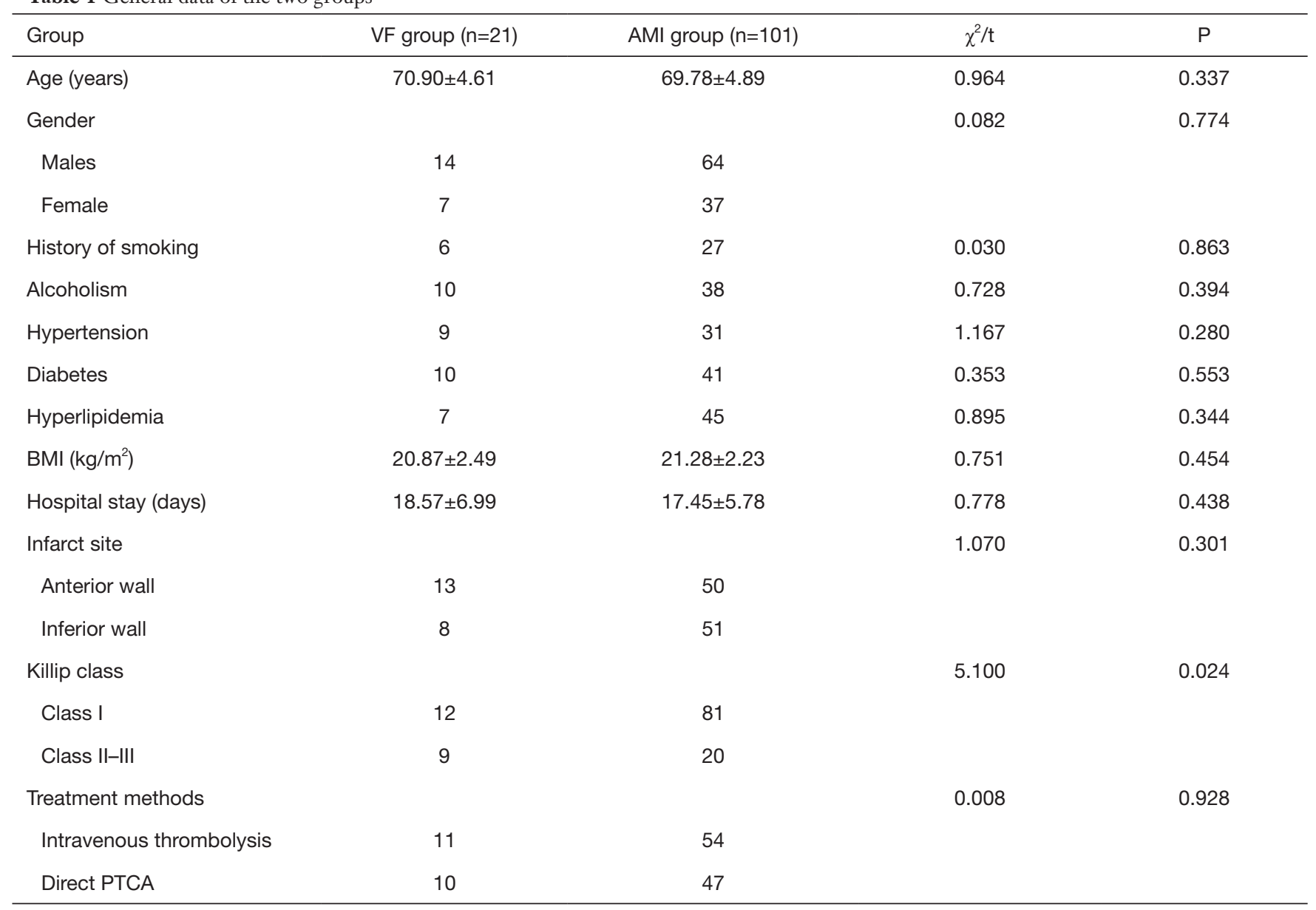

VF, ventricular fibrillation; AMI, acute myocardial infarction; PTCA, percutaneous coronary angioplasty.

successive differences of normal RR intervals (rMSSD), and percentage of $\mathrm{NN} 50$ in the total number of $\mathrm{NN}$ intervals PNN50 (PNN50\%) were recorded.

\section{Statistical analysis}

Statistical analysis was performed by using the SPSS 20.0 software package. Measurement data are presented as mean \pm standard deviation $(\bar{x} \pm S D)$ and were compared using independent samples $t$-test, and the count data were analyzed using Chi-square test. The receiver operating characteristic (ROC) curves were used to evaluate the predictive value of HRV for elderly patients with AMI complicated by VF. The comparisons of area under the ROC curves (AUC) were based on the $\mathrm{Z}$ score test. A P value of $<0.05$ was considered statistically significant.

\section{Results}

\section{AMI complicated with VF in elderly patients}

Results showed that $17.21 \%(21 / 122)$ of elderly AMI patients experienced VF during hospitalization.

\section{General data in two groups}

The two groups had no statistically significant differences in age, gender, alcoholism, smoking history, hypertension, diabetes, hyperlipidemia, body mass index (BMI), hospital stay, infarct site, and treatment methods (all $\mathrm{P}>0.05$ ); however, Killip class showed significant differences between the groups $(\mathrm{P}<0.05)$ (Table 1).

Laboratory findings in two groups 
Table 2 Laboratory findings in the two groups

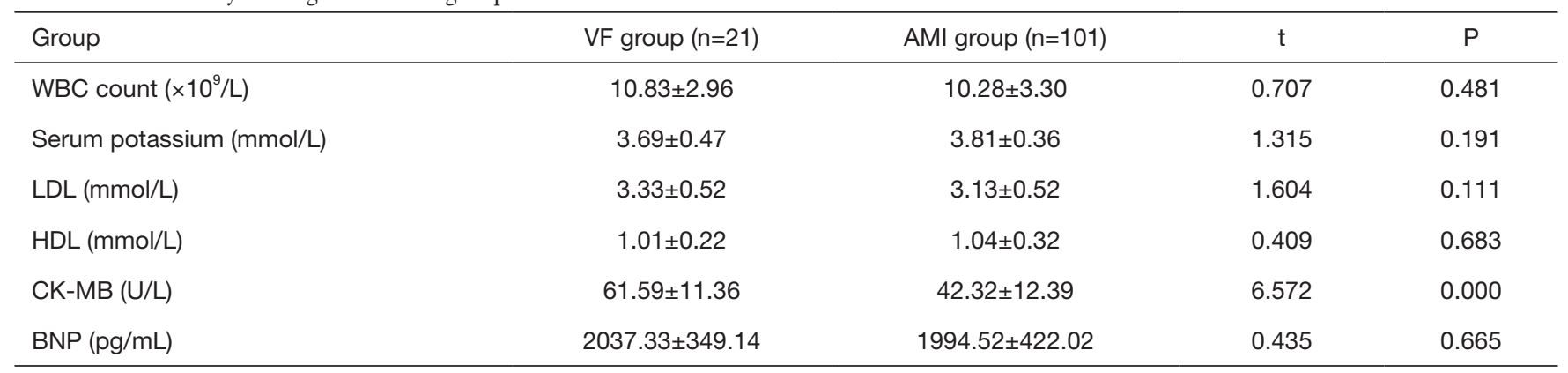

VF, ventricular fibrillation; AMI, acute myocardial infarction; LDL, low-density lipoprotein; HDL, high-density lipoprotein; CK-MB, creatine kinase myocardial band; BNP, brain natriuretic peptide.

Table 3 Comparison of HRV indexes between the two groups $(\bar{x} \pm \mathrm{SD})$

\begin{tabular}{lccc}
\hline Group & VF group $(\mathrm{n}=21)$ & AMl group $(\mathrm{n}=101)$ & $\mathrm{t}$ \\
\hline SDNN (ms) & $110.80 \pm 21.38$ & $136.49 \pm 27.67$ & 4.008 \\
SDANN (ms) & $101.74 \pm 28.22$ & $126.14 \pm 22.67$ & 4.295 \\
rMSSD (ms) & $28.86 \pm 7.10$ & $38.72 \pm 8.87$ & 0.001 \\
PNN50\% & $9.31 \pm 3.98$ & $15.27 \pm 5.17$ & 4.780 \\
\hline
\end{tabular}

HRV, heart rate variability; VF, ventricular fibrillation; AMI, acute myocardial infarction; AUC, area under the ROC curve; SDNN, standard deviation of all R-R intervals; SDANN, standard deviation of the R-R intervals; rMSSD, root-mean-square of the successive differences of normal RR intervals; PNN50\%, percentage of NN50 in the total number of NN intervals PNN50.

White blood cell count and serum levels of potassium, lowdensity lipoprotein (LDL), high-density lipoprotein (HDL), and brain natriuretic peptide (BNP) were not significantly different between the VF group and the AMI group after admission (all $\mathrm{P}>0.05$ ); however, the $\mathrm{VF}$ group had significantly higher activity of creatine kinase myocardial band $(\mathrm{CK}-\mathrm{MB})$ isoenzyme than the AMI group $(\mathrm{P}<0.05)$ (Table 2).

\section{Comparison of HRV indexes between the two groups}

SDNN, SDANN, rMSSD, and PNN50\% were significantly higher in the AMI group than in the $\mathrm{VF}$ group $(\mathrm{P}<0.05)$ (Table 3).

\section{Predictive value of HRV indexes in elderly patients with AMI complicated with $V F$}

The ROC curves were used to analyze the predictive values of SDNN, SDANN, rMSSD, and PNN50\% for elderly patients with AMI complicated by VF. The $\mathrm{ROC}_{\mathrm{AUC}}$ of SDNN, SDANN, rMSSD, and PNN50\% was $0.773,0.761$,
0.800 , and 0.819 , respectively, $(\mathrm{P}>0.05)$ and the cutoff values were $135.675,106.850,33.570$, and 14.080 , respectively (Figure 1 and Table 4).

\section{Discussion}

AMI is a common clinical heart disease and typically manifests clinically as pain behind the sternum that cannot be medically relieved. As a fast-onset condition, AMI is one of most fatal diseases for human beings. Clinically, AMI is often treated with percutaneous coronary intervention to achieve rapid recovery (9). Up to $80 \%$ of AMI patients have different degrees of arrhythmia, which typically occur 24-48 h after disease onset. Ventricular arrhythmia (especially the complicated VF) is the most common arrhythmia type in AMI patients. Thus, early detection and early treatment are essential for improving the outcomes of these patients. VF is one of the most severe complications of AMI and also the main cause of death in AMI patients (10-12). The incidence of VF shows an upward trend in the elderly population (13), likely because degenerative alterations of the body occur with age, and most elderly patients have underlying diseases 
such as hypertension and diabetes, which further increase the risk of VF. Therefore, an effective predictive index for AMI complicated with VF is particularly important for reducing the risk of VF in elderly AMI patients. In our current study, 122 elderly AMI patients admitted to our hospital were selected as the subjects, and VF was detected in 21 patients $(17.21 \%)$ during their hospitalization. The proportion of Killip class II/III and the activity of CK-MB were significantly higher in the VF group than in the AMI group. We thus speculate that cardiac injury is more severe in elderly AMI patients complicated by VF than in those with simple AMI.

The electrical stability of cardiomyocytes can be controlled by body fluids and autonomic nerves. Cardiac autonomic nerves include the vagus nerve and the

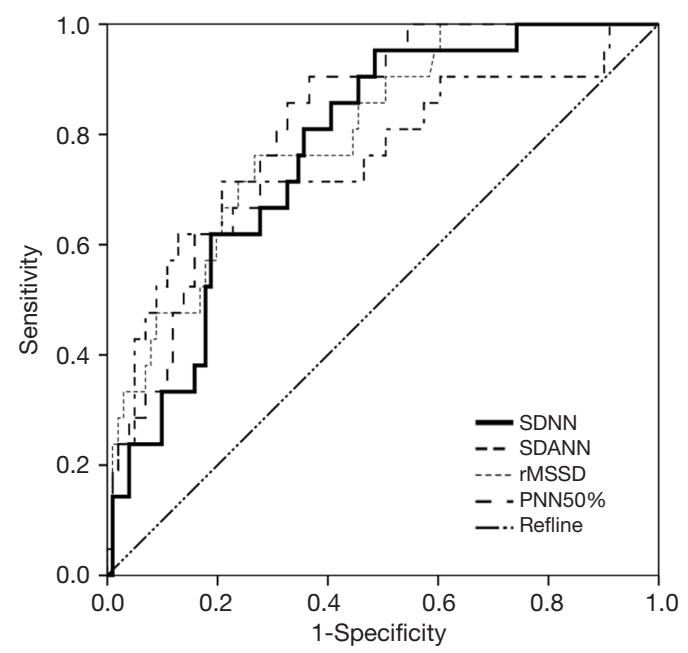

Figure 1 ROC curve values of heart rate variability indices in predicting $\mathrm{VF}$ in elderly $\mathrm{AMI}$ patients. ROC, receiver operating characteristic; VF, ventricular fibrillation; AMI, acute myocardial infarction. sympathetic nerve, and both can regulate the heart. Decrease in the regulatory function of the vagus nerve will increase the incidence of arrhythmia (14). The vagus nerve has a stimulating effect on acetylcholine, and it can also regulate the negative conduction, frequency of contraction, and muscle strength of the heart through reflexes. It was previously believed that cardiovascular events such as arrhythmia, cardiac death, and sudden death were mainly related to increased sympathetic excitability; however, recent studies have shown that these events are linked to the decreased protective ability of the vagus nerve (15). Some authors claim that the innervation abilities of the vagus nerve and sympathetic nerve are unequal, stating that the vagus nerve has a stronger regulating ability during a resting state, and its regulatory ability is weakened in the exercise state, leading to an elevated heart rate (16). AMI patients experience symptoms like myocardial ischemia, necrosis, and remodeling, in addition to ventricular dilation and fibrosis, which cause damage to the distal ends of receptors and break the balance of cardiac autonomic nerves; as a result, the regulatory abilities of heart tissues decrease, the activity of the vagus nerve and the VF threshold declines, and the electrical stability of cardiomyocytes is reduced (17).

HRV is a highly useful quantitative indicator in evaluating the function of cardiac autonomic nerves. It can be used as one of the key predictive indicators of arrhythmia events and sudden cardiac death and is also highly valuable in diagnosing cardiovascular disease. The HRV time-domain indices include SDNN, SDANN, rMSSD, and PNN50\%. SDNN, as a normal response of the heart to multiple physiological and environmental stimuli, reflects the heart rate and HRV. It can indicate a patient's autonomic nerve function and the degree of damage. SDANN can be used to evaluate sympathetic tone, which reflects the functional state of the sympathetic nerve. Research has shown SDANN has a negative correlation

Table 4 Performance of heart rate variability indices in predicting VF in elderly AMI patients

\begin{tabular}{llcccc}
\hline Index & AUC & SE & Cutoff value & $\mathrm{P}$ & $95 \% \mathrm{Cl}$ \\
\hline SDNN (ms) & 0.773 & 0.049 & 135.675 & 0.000 & $0.677-0.869$ \\
SDANN (ms) & 0.761 & 0.066 & 106.850 & 0.000 & $0.632-0.890$ \\
rMSSD (ms) & 0.800 & 0.049 & 33.570 & 0.000 & $0.704-0.897$ \\
PNN50\% & 0.819 & 0.043 & 14.080 & 0.000 & $0.736-0.903$ \\
\hline
\end{tabular}

VF, ventricular fibrillation; AMI, acute myocardial infarction; AUC, area under the ROC curve; SDNN, standard deviation of all R-R intervals; SDANN, standard deviation of the R-R intervals; rMSSD, root-mean-square of the successive differences of normal RR intervals; PNN50\%, percentage of NN50 in the total number of NN intervals PNN50. 
with sympathetic function (18). rMSSD and PNN5\% can reveal the sensitivity of vagus nerve to stimuli, and low rMSSD and PNN5\% values imply the decreased excitation of the vagus nerve (19). Increased levels of SDNN, SDANN, rMSSD, and PNN50\% indicate an increased parasympathetic tone and raised VF threshold; in contrast, decreases in these indices indicate that the sympathetic tone is increased and the VF threshold is declined $(20,21)$. As shown in our current study, the SDNN, SDANN, rMSSD, and $\mathrm{PNN} 50 \%$ were significantly lower in the $\mathrm{VF}$ group than in the AMI group; meanwhile, their AUCs were 0.773, $0.761,0.800$, and 0.819 , respectively, and the cutoff values were $135.675,106.850,33.570$, and 14.080 , respectively, suggesting HRV has certain predictive value for elderly patients with AMI complicated by VF.

In summary, the cardiac injury is more severe in elderly AMI patients complicated by VF than in those with simple AMI. HRV has a certain predictive value for elderly patients with AMI complicated by VF. HRV changes on dynamic electrocardiography should be constantly monitored for elderly AMI patients within $24 \mathrm{~h}$ after admission, and timely intervention measures should be taken when VF occurs.

\section{Acknowledgments}

Funding: None.

\section{Footnote}

Reporting Checklist: The authors have completed the STROBE reporting checklist. Available at http://dx.doi. org/10.21037/apm-20-1362

Data Sharing Statement: Available at http://dx.doi. org/10.21037/apm-20-1362

Conflicts of Interest: All authors have completed the ICMJE uniform disclosure form (available at http://dx.doi. org/10.21037/apm-20-1362). The authors have no conflicts of interest to declare.

Ethical Statement: The authors are accountable for all aspects of the work in ensuring that questions related to the accuracy or integrity of any part of the work are appropriately investigated and resolved. The study was approved by the ethics committee of our hospital (No. 2018010). All the patients attended the study voluntarily. Patients and their families signed informed consent documents. The study was conducted in accordance with the Declaration of Helsinki (as revised in 2013).

Open Access Statement: This is an Open Access article distributed in accordance with the Creative Commons Attribution-NonCommercial-NoDerivs 4.0 International License (CC BY-NC-ND 4.0), which permits the noncommercial replication and distribution of the article with the strict proviso that no changes or edits are made and the original work is properly cited (including links to both the formal publication through the relevant DOI and the license). See: https://creativecommons.org/licenses/by-nc-nd/4.0/.

\section{References}

1. Guo W, Yang D, Wu D, et al. Hyperuricemia and longterm mortality in patients with acute myocardial infarction undergoing percutaneous coronary intervention. Ann Transl Med 2019;7:636.

2. Nichol G, Sayre MR, Guerra F, et al. Defibrillation for Ventricular Fibrillation: A Shocking Update. J Am Coll Cardiol 2017;70:1496-509.

3. Heusch G, Gersh BJ. The pathophysiology of acute myocardial infarction and strategies of protection beyond reperfusion: a continual challenge. Eur Heart J 2017;38:774-84.

4. Rizos T, Güntner J, Jenetzky E, et al. Continuous stroke unit electrocardiographic monitoring versus 24-hour Holter electrocardiography for detection of paroxysmal atrial fibrillation after stroke. Stroke 2012;43:2689-94.

5. Kim HG, Cheon EJ, Bai DS, et al. Stress and Heart Rate Variability: A Meta-Analysis and Review of the Literature. Psychiatry Investig 2018;15:235-45.

6. Song T, Qu XF, Zhang YT, et al. Usefulness of the heartrate variability complex for predicting cardiac mortality after acute myocardial infarction. BMC Cardiovasc Disord, 2014;14:59

7. Stengaard C, Sørensen JT, Rasmussen MB, et al. Prehospital diagnosis of patients with acute myocardial infarction. Diagnosis (Berl) 2016;3:155-66.

8. Cao X, Müller A, Dirschinger RJ, et al. Risk Prediction After Myocardial Infarction by Cyclic Variation of Heart Rate, a Surrogate of Sleep-Disordered Breathing Assessed From Holter ECGs. Front Physiol 2020;10:1570.

9. January CT, Wann LS, Alpert JS, et al. 2014 AHA/ACC/ HRS guideline for the management of patients with atrial fibrillation: a report of the American College of Cardiology/American Heart Association Task Force on 
Practice Guidelines and the Heart Rhythm Society. J Am

Coll Cardiol 2014;64:e1-e76.

10. Zhang $\mathrm{Y}$, Cao H, Jiang $\mathrm{P}$, et al. Cardiac rehabilitation in acute myocardial infarction patients after percutaneous coronary intervention: A community-based study. Medicine (Baltimore) 2018;97:e9785.

11. Kosmidou I, Embacher M, McAndrew T, et al. Early Ventricular Tachycardia or Fibrillation in Patients With ST Elevation Myocardial Infarction Undergoing Primary Percutaneous Coronary Intervention and Impact on Mortality and Stent Thrombosis (from the Harmonizing Outcomes with Revascularization and Stents in Acute Myocardial Infarction Trial). Am J Cardiol 2017;120:1755-60.

12. Lüscher TF. Supraventricular and ventricular tachycardias: risk factors, drugs, and ablation. Eur Heart J 2017;38:1271-4.

13. Jabbari R. Ventricular fibrillation and sudden cardiac death during myocardial infarction. Dan Med J 2016;63:B5246.

14. Masuda M, Nakatani D, Hikoso S, et al. Clinical Impact of Ventricular Tachycardia and/or Fibrillation During the Acute Phase of Acute Myocardial Infarction on InHospital and 5-Year Mortality Rates in the Percutaneous Coronary Intervention Era. Circ J 2016;80:1539-47.

15. Lin TT, Yang WS, Hsieh MY, et al. Deterioration of Deceleration Capacity of Heart Rate is Associated with Left Ventricular Hypertrophy in End-Stage Renal Disease Population. Acta Cardiol Sin 2018;34:242-50.

Cite this article as: Zhang Y, Wang J, Xu Y. Value of heart rate variability on dynamic electrocardiogram in predicting ventricular fibrillation in elderly acute myocardial infarction patients. Ann Palliat Med 2020;9(5):3488-3494. doi: 10.21037/ apm-20-1362
16. Bianchi S, Rossi P, Schauerte P, et al. Increase of ventricular interval during atrial fibrillation by atrioventricular node vagal stimulation: chronic clinical atrioventricular-nodal stimulation download study. Circ Arrhythm Electrophysiol 2015;8:562-8.

17. Gold MR, Van Veldhuisen DJ, Hauptman PJ, et al. Vagus Nerve Stimulation for the Treatment of Heart Failure: The INOVATE-HF Trial. J Am Coll Cardiol 2016;68:149-58.

18. Wang YP, Kuo TB, Li JY, et al. The relationships between heart rate deceleration capacity and spectral indices of heart rate variability during different breathing frequencies. Eur J Appl Physiol 2016;116:1281-7.

19. Katz-Leurer M, Zohar N, Boum A, et al. Monitoring changes in heart rate, as an indicator of the cardiovascular autonomic nervous function, among patients at the subacute phase post-brain damage during a physiotherapy session: a preliminary investigation. Brain Inj 2014;28:127-31.

20. Pinter A, Horvath T, Sarkozi A, et al. Relationship between heart rate variability and endothelial function in healthy subjects. Auton Neurosci 2012;169:107-12.

21. Lu DY, Yang AC, Cheng HM, et al. Heart Rate Variability Is Associated with Exercise Capacity in Patients with Cardiac Syndrome X. PLoS One 2016;11:e0144935.

(English Language Editor: J. Gray) 